Documentation et bibliothèques

DOCUMENTATION BIBLIOTHÈQUES

\title{
Le système Esar : modalités d'application et traitement documentaire informatisé de jeux et jouets à la Centrale des bibliothèques du Québec
}

\section{Denise Garon}

Volume 29, numéro 1, janvier-mars 1983

URI : https://id.erudit.org/iderudit/1053645ar

DOI : https://doi.org/10.7202/1053645ar

Aller au sommaire du numéro

Éditeur(s)

Association pour l'avancement des sciences et des techniques de la documentation (ASTED)

ISSN

0315-2340 (imprimé)

2291-8949 (numérique)

Découvrir la revue

Citer cet article

Garon, D. (1983). Le système Esar : modalités d'application et traitement documentaire informatisé de jeux et jouets à la Centrale des bibliothèques du Québec. Documentation et bibliothèques, 29(1), 35-40.

https://doi.org/10.7202/1053645ar

Tous droits réservés (C) Association pour l'avancement des sciences et des techniques de la documentation (ASTED), 1983
Ce document est protégé par la loi sur le droit d'auteur. L'utilisation des services d'Érudit (y compris la reproduction) est assujettie à sa politique d'utilisation que vous pouvez consulter en ligne.

https://apropos.erudit.org/fr/usagers/politique-dutilisation/ 


\section{Le système Esar: Modalités d'application et traitement documentaire informatisé de jeux et jouets à la Centrale des bibliothèques du Québec}

\section{Introduction}

Au Québec, la recherche orientée spécifiquement vers les services à l'enfance s'est trop souvent laissée dépasser par les événements en venant confirmer ou infirmer trop tard le bienfondé d'expériences nouvelles ou en offrant parfois après-coup les instruments psychopédagogiques dont le milieu a besoin.

L'implantation chez-nous de services de prêts de jouets et de matériel de jeu appelés joujouthèques ou ludothèques, fait appel à un esprit novateur de mise en commun de ressources éducatives et suscite en même temps, de nombreuses interrogations. Les responsables de tels services doivent en effet pouvoir analyser et classer un grand nombre d'objets de toutes sortes d'une manière logique et efficace tout en tenant compte des dimensions éducatives généralement véhiculées par des objets à caractère ludique.

Dans une telle perspective, pour répondre à la fois aux besoins d'analyse psychologique du matériel ludique et à la nécessité de regrouper et de classer de façon méthodique tous ces accessoires de jeux, une recherche doctorale entreprise à la faculté des Sciences de l'éducation de I'Université Laval, a tenté d'associer, dans une même démarche, le langage psychologique et le langage des techniques documentaires. Le cadre théorique défendu dans la première partie de cette thèse a permis d'élaborer un modèle de classement dont les grandes caractéristiques sont inspirées du schéma de classification à facettes. Le modèle de classement ESAR est constitué en effet de facettes complémentaires représentant divers aspects du savoir-jouer. Le nom même du modèle est tiré du premier volet du système. II correspond aux grandes catégories de jeux inspirées de l'approche "piagétienne» et couvre l'ensemble du développement de l'activité ludique, de la petite enfance à l'âge adulte: ce sont les jeux d'exercice (E). les jeux symboliques (S), les jeux d'assemblage (A) et les jeux de règles simples et de règles complexes (R). Les modalités d'application de la recherche théorique ont fait l'objet d'un projet concret mené à la Centrale des bibliothèques. Le rapport détaillé de cette application dans le milieu est présenté dans la deuxième partie de la thèse.

\section{Historique du projet}

Au cours de l'année 1979-1980, douze ludothèques implantées un peu partout au Québec. dans le cadre des politiques d'éducation en milieu économiquement faible ${ }^{1}$, ont monté diverses collections de jeux et de jouets destinés à être prêtés à des groupes d'enfants de quatre et de cinq ans, à l'aide d'un guide d'organisation des ludothèques ${ }^{2}$. A cause du nombre grandissant de parents-usagers bénévoles et de la diversité des services offerts (dépôts, collections itinérantes. etc.). le ministère de l'Éducation confiait, à la fin de l'automne 1980, un mandat de travail à la Centrale des bibliothèques ${ }^{3}$, pour assumer la codification, la classification et l'analyse documentaire du matériel ludique destiné à être utilisé de façon très large, par les ludothèques et par de nombreux éducateurs.

Pour mener à bien son projet, la Centrale des bibliothèques a conclu au début de 1981, des ententes avec la bibliothèque publique de PointeClaire en banlieue de Montréal et avec le Centre d'information sur la santé de l'enfant (CISE) de l'hôpital Sainte-Justine de Montréal, pour l'emprunt de jouets et de matériel de jeu et pour une association de ressources. Elle a en outre bénéficié de la collaboration de la bibliothèque municipale de Montréal, de la BCP de l'Outaouais, de la Joujouthèque de Sainte-Foy, etc. Les responsables du projet ont, dans le même sens, retenu la méthode de classement ESAR comme support d'analyse spécifique des contenus ludiques à

1 Québec, L'école québécoise: Énoncé de polıtıque et plan d'action, Québec. Éditeur officiel du Québec, 1979

2. Québec, Guide d'organisation d'une ludothèque, Québec, Direction générale des réseaux, ministère de l'Éducation. 1979

3. Il convient ici de souligner l'appui et le soutien apportés par l'équipe de la Centrale des bibliothèques et en particulier la collaboration de Jacques Demers et Marielle Boucher. respectivement responsable et coordonnateur du «projet ludothèques "; de Marie-Claude Pıquion, éducatrice à l'hôpital Ste-Justine et de Rolande Filion, responsable de la joujoutheque de Sainte-Foy, toutes deux analystes de jouets; de monsieur Alain Boucher, directeur de la planification et du développement à la Centrale; de Jean-Claude Racicot, informaticien et de Mireille Massy. responsable de la saısıe des données. L'intérêt et le support apportés par les membres du comıté consultatıf ont également été très appréciés de même que l'appui soutenu de Marie Giroux. responsable du dossier ludothèques au ministère de l'Éducation. 


\section{Plan d'ensemble méthodique des concepts psychopédagogiques constituant la banque de descripteurs dans le modèle de classement ESAR}

FACETTE A

\section{ACTIVITESS LUDIQUES}

1. JEU D'EXERCICE

$$
\begin{aligned}
& \text { 01. Jeu sensoriel sonore } \\
& \text { 02. Jeu sensoriel visuel } \\
& \text { 03. Jeu sensoriel tactile } \\
& \text { 04. Jeu sensortel olfactif } \\
& \text { 05. Jeu sensoriel gustatif } \\
& \text { 06. Jeu moteur } \\
& \text { 07. Jeu de manipulation }
\end{aligned}
$$

2. JEU SYMBOLIQUE

$$
\begin{aligned}
& \text { 01. Jeu de falre-semblant } \\
& \text { 02. Jeu de rôles } \\
& \text { 03. Jeu de représentation }
\end{aligned}
$$

3. JEU D'ASSEMBLAGE

$$
\begin{aligned}
& \text { 01. Jeu de construction } \\
& 02 \text {. Jeu d'agencement } \\
& \text { 03. Jeu de montage mécanique } \\
& 04 \text {. Jeu de montage électro-mécanique } \\
& \text { 05. Jeu de montage électronique } \\
& \text { 06. Jeu d'assemblage scientifique } \\
& \text { 07. Jeu d'assemblage artistique }
\end{aligned}
$$

4. JEU DE RÈGLES SIMPLES

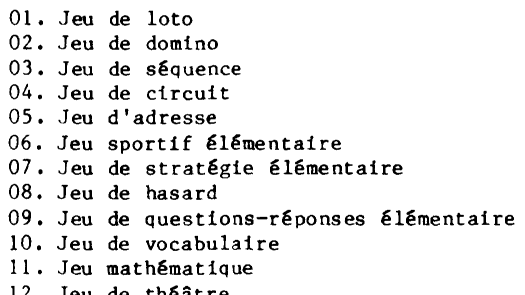

12. Jeu de thêatre

5. JEU DE REGLES COMPLEXES

01. Jeu de réflexion

02. Jeu sportif complexe

03. Jeu de stratégie complexe

04. Jeu de hasard

05. Jeu de questions-réponses complexe

06. Jeu de vocabulaire complexe

07 . Jeu d'analyse mathematique

08. Jeu d'assemblage complexe

09. Jeu de représentation complexe

10. Jeu de scène
FACETTE B

CONDUITES COGNITIVES

1. CONDUITE SENSORI-MOTRICE
01. Répétition
02 . Reconnaissance sensori-motrice
03. Généralisation sensori-motrice
04. Raisonnement pratique

2. CONDUITE SYMBOL IQUE
01 . Evocation symbolique
02. Lialsons images-mots
03. Expression verbale
04. Pensée représentat ive

3. Conduite intuitive

$$
\begin{aligned}
& \text { 01. Triage } \\
& \text { 02. Apparlement } \\
& \text { 03. Différenciation de couleurs } \\
& \text { 04. Différenciation de dimenstons } \\
& \text { 05. Différenciation de formes } \\
& \text { 06. Différenciation de textures } \\
& \text { 07. Différenciation temporelle } \\
& \text { 08. Différenciation spatiale } \\
& \text { 09. Association d'idées } \\
& \text { 10. Rafsonnement intuitif }
\end{aligned}
$$

4. CONDUITE OPERATOIRE CONCRËTE

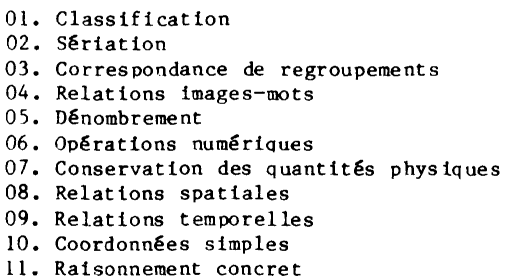

5. CONDUITE OPERATOIRE FORMELLE
01. Raisonnement hypothétique
02. Raisonnement deduct tf
03. Ra1 sonnement' Induct if
04. Ralsonnement combinatoire
05 . Système de représentations complexes
06. Système de coordonnées complexes

répertorier. Le «projet ludothèques » mis sur pied par la Centrale des bibliothèques constitue donc. dans les faits, le milieu d'application de la recherche doctorale entreprise à l'Université Laval.

\section{Particularités du système ESAR}

Le modèle de classement ESAR a été conçu pour faciliter et systématiser la désignation de descripteurs pertinents, adaptés à une réalité psychologique précise et hiérarchisée: il se présente dans sa forme actuelle comme un ensemble de quatre facettes initiales correspondant à quatre dimensions complémentaires du savoir-jouer: la première facette désigne les familles d'activités ludiques fondamentales, c'est-à-dire les jeux d'exercice, de symbole, d'assemblage et de règles: la facette des conduites cognitives témoigne des niveaux de complexité mentale des activités ludi- 
FACETTE C

HABILETÉS FONCTIONNELLES

1. EXPLORATION

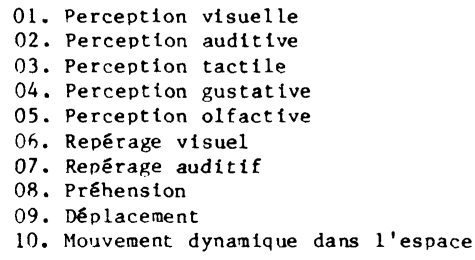

2. IMITATION

01. Reproduction d'actions

02. Reproduction d'objets

03. Reproduction d'événements

04. Reproduction de rôles

05 . Reproduction de modèles

06. Reproduction de mots

07. Reproduction de sons

08 . Application de règles

09. Attention visuelle

10. Attention auditive

11. Discrimination visuelle

12. Discrimination auditive

13. Discrimination tactile

14. Discrimination gustative

15. Discrimination olfact tue

16. Mémolre visuelle

17. Mémoire auditive

18. Mémolre tactlle

19. Mémolre gustative

20. Mémotre olfactive

21. Coordination oeil-main

22 . Coordination oet1-pied

23. Orfentation spatiale

24. Orientation temporelle

25. Organisation spatiale

26. Organisation temporelle

3. PERFORMANCE
01. Acuite visuelle
02. Acuité auditive
03. Dextérité
04. Souplesse
05. Ag1lité
06. Endurance
07. Force
08. Rapidite
09. Précision
10. Patience
11. Concentration
12. Mémo1re logique

4. CREATION

01. Créativité d'expression

02. Créativité productive

03. Créativité inventive
FACETTE D

ACTIVITÉS SOCIALES

1. ACTIVITE INDIVIDUELLE

01. Activité solitaire

02. Activité parallèle

2. PARTICIPATION COLLECTIVE

01. Activité as sociative

02. Activité compétitive

03. Activité coopérative

3. PARTICIPAT:ON VARIABLE

01. Activité solltaire ou parallèle

02. Activité solitaire ou associative

03. Activite solitaire ou compétitive

04. Activité solitaire ou cooperative ques: la facette des habiletés fonctionnelles met en évidence les types d'habiletés nécessaires pour jouer et la facette sociale distingue les formes de participation possibles au cours des activités de jeu. La possibilité de pouvoir ajouter ultérieurement deux autres facettes complémentaires dans le même esprit d'analyse méthodique. c'est-à-dire la dimension du langage et l'expression des émotions, fait partie de la stratégie générale de conception du système.
Le modèle de classement ESAR permet, à l'étape d'indexation, dans une démarche de traitement documentaire, de représenter de façon méthodique, les contenus psychologiques du savoir-jouer, à l'aide de mots-clés spécifiques. Le plan d'ensemble de ces mots-clés constitue en quelque sorte le thésaurus où va puiser l'analyste. Chacun des aspects complémentaires du savoir-jouer représente une facette particulière. Les dimensions fondamentales de chacun des 
aspects retenus forment des catégories générales à l'intérieur des facettes et portent le nom de descripteurs principaux. Les dimensions plus spécifiques forment des sous-catégories à l'intérieur des facettes et deviennent des descripteurs secondaires. Ces répertoires et sous-répertoires de mots-clés sont agencés de façon cumulative et hiérarchique et les croisements innombrables auxquels ils peuvent donner lieu contribuent à préciser les caractéristiques des grandes activités de jeu et celles des accessoires qui les accompagnent. Pour rendre fonctionnelle l'utilisation de ce plan théorique, chaque descripteur est identifié par une cote alphanumérique (tableau) et chacun des concepts contenus sous chaque descripteur est défini avec précision de manière à rendre les jugements des analystes plus objectifs. Un fichier de définitions de l'ensemble des concepts accompagne donc l'application du système. Les principales assises théoriques du système ont fait l'objet d'un article paru dans un numéro antérieur de Documentation et bibliothèques ${ }^{4}$. De plus, l'ensemble de la démarche est présenté en détails, dans le texte intégral de la thèse doctorale déposée à l'Université Laval de Québec 5

\section{Modalités d'application de la méthode}

Le «projet ludothèques», tel qu'envisagé par la Centrale des bibliothèques du Québec, prévoit dans sa démarche le traitement informatisé de toutes les données obtenues au cours de l'analyse documentaire, l'enregistrement et la conservation de toutes les notices bibliographiques sur mémoire informatisée. Cet organisme dispose en effet dans ses locaux, depuis février 1981, d'un ordinateur Hewlett-Packard, série 3000, modèle 111 entièrement réservé à la réalisation de travaux et de projets documentaires de nature variée.

Pour rentabiliser au maximum ses équipements techniques, la Centrale a choisi d'associer au logiciel d'exploitation propre à cet ordinateur, un progiciel d'application plus spécifique: le progiciel documentaire MINISIS, mis au point et développé par le Centre de recherches pour le développement international (CRDI) à Ottawa. MINISIS est un système de gestion et de recherche de l'information conçu pour fonctionner sur des ordinateurs Hewlett-Packard de la série 3000. dans le but de répondre au besoin d'un ensemble matériel-logiciel n'exigeant qu'un investissement modéré: il permet l'entrée de données et la

4. Denıse Garon-Dupont, "Le système ESAR: un concept nouveau de classification des jouets à l'intention des ludothécaires». Documentation et bibliothèques, vol. 25. no 4 (décembre 1979), 189-199.

5. Denise Garon. Le système ESAR: Un modèle de classement des jouets et du matériel de jeu à l'intention des éducateurs, Québec, Université Laval. Thèse de doctorat inédite. 295 pages. recherche interactive en direct et ne présente pas de difficultés pour l'utilisateur-chercheur, même pour celui qui n'a pas d'expérience en matière de système d'information automatisée : la consultation en mode conversationnel permet en effet d'avoir accès, dans un langage simple, à un ensemble de données bibliographiques consignées dans le système informatisé.

MINISIS comprend un jeu de programmes destinés à la gestion des bibliothèques et à la recherche documentaire: son processeur de recherche dispose d'une puissante capacité de récupération pour soumettre, modifier ou rechercher des données et pour assurer le traitement complet de séries d'informations à partir de thésaurus, et la reconnaissance multilingue des termes de ce thésaurus. II peut également produire des bibliographies annotées, des catalogues de bibliothèques et différents types d'index.

À partir des multiples possibilités d'un tel support matériel, les responsables du projet ont préparé un plan de codage et déterminé les zones d'informations à retenir pour constituer une base de données significative et pertinente, adaptée au domaine d'analyse du jeu et du jouet. L'ensemble des codes mnémoniques permettant de rédiger des notices bibliographiques convenant à l'analyse documentaire des jeux et jouets, est contenu dans le répertoire des zones et des souszones des notices de la base de données et sert de clé de voûte à l'ensemble de la démarche informatisée.

Le répertoire des zones et des sous-zones des notices de la base de données a été préparé suivant l'ordre logique des étapes principales d'analyse du matériel de jeu. Il permet ainsi de coder avec le nom de la zone, l'indicatif mnémonique et un numéro d'étiquette, des informations particulières au catalogue descriptif. Il code de la même manière, toutes les composantes méthodiques du système de classement ESAR. Le répertoire prévoit également une zone d'annotation en trois volets permettant d'enregister l'analyse du document ludique, les commentaires spécifiques et la recommandation d'âge des usagers.

\section{Travail des analystes}

Les instruments mis au service des analystes du jeu ont été réunis grâce au travail complémentaire des responsables du projet, de la technicienne en catalogage, de l'auteur du modèle de classement ESAR et des avis du comité consultatif. Compte tenu du caractère inédit de la démarche entreprise, le mandat des analystes comportait une large part d'utilisation critique des instruments de travail, de manière à raffiner ces instruments à partir d'une première utilisation. En plus de la collection des objets à analyser, les analystes ont travaillé à l'aide de plusieurs éléments. 
En premier lieu, un guide d'analyse des jouets et du matériel de jeu 6 regroupe l'essentiel des informations nécessaires: ce document de travail présente les principes et modalités d'application du système ESAR, le protocole d'analyse, le plan de classification et la grille de 151 descripteurs ou mots-clés tenant lieu de thésaurus. De plus, le guide contient les formules de description et d'évaluation du matériel de même que le bordereau d'indexation, le bordereau d'entrée des données et un exemple de fiche bibliographique complétée en version de travail. Plusieurs éléments du guide sont tirés des deux premiers chapitres de la thèse; ces éléments ont en effet été décrits comme étant des assises théoriques servant de toile de fond, sur le plan psychopédagogique au modèle de classement ESAR: il est donc normal qu'ils se retrouvent au coeur même du guide mis à la disposition des analystes utilisant le système. De plus, plusieurs versions successives ont été nécessaires pour réunir l'ensemble des informations pertinentes.

Le plan méthodique de classification a été proposé et justifié dans la première partie de la recherche. Ce plan de classification a été transformé en thésaurus spécialement adapté à l'analyse des objets ludiques. Ce répertoire de termes normalisés en vue de l'analyse de contenus spécifiques, sert à la fois de grille d'analyse et de banque de descripteurs. Un code alphanumérique a été appliqué à la banque de descripteurs et permet de procéder selon une démarche systématique à l'analyse des objets de jeu (tableau).

Un fichier de définitions accompagne le guide d'analyse. Ce fichier permet d'uniformiser le sens précis donné à chacun des concepts servant de descripteurs principaux et secondaires retenus pour constituer la banque de descripteurs.

\section{Utilisations possibles et perspectives d'avenir}

La Centrale des bibliothèques, dès le début du "projet ludothèques», avait pour objectif de diffuser les résultats des travaux d'analyses documentaires appliqués à dès collections de jeux, de jouets et de matériel de jeu. II y a quelques mois, les responsables du projet ont offert à d'éventuels abonnés, la publication d'une revue portant sur les jeux et jouets. En préparant ChoixJeunesse: Jeux et jouets, la Centrale tentait de rejoindre tout un groupe d'éducateurs, de parents et de professionnels de l'activité ludique. La réponse des usagers a été fort éloquente: plus de 2000 demandes ont été reçues pour le premier numéro qui est distribué gratuitement dans sa version photocomposée.

Le Choix-Jeunesse: Jeux et jouets se présente comme une série de notices distribuées sur deux colonnes, avec photographies des objets analysés. Le premier numéro propose 130 analyses, le second en comprend 235 . Ils sont disponibles en édition photocomposée et en édition imprimée sur des cartons de couleurs (une couleur différente pour chaque grande division de la facette $A$ du système ESAR).

Ce répertoire de jeux et jouets présente en plus du plan d'ensemble des descripteurs du système ESAR différents index: noms de fabricants ou de créateurs d'objets; titres et noms des objets anaIysés; séries, collections et descripteurs. De plus. ces analyses sont regroupées selon un ordre correspondant aux quatre principales familles ou groupes de jeux du système ESAR, c'est-à-dire les jeux d'exercice, les jeux symboliques, les jeux d'assemblage et les jeux de règles simples complexes.

Les projets à long terme sont pour une bonne part liés à la forme d'intérêt déjà présent dans le milieu et par ailleurs rattachés aussi aux diverses possibilités de recherches suscitées par la présente démarche d'analyse. II faudra donc continuer d'alimenter la base de données et analyser périodiquement du nouveau matériel. La Centrale espère obtenir les budgets nécessaires pour continuer le travail amorcé.

La Centrale a également publié en coédition une bibliographie annotée de monographies, d'articles de périodiques et de documents audiovisuels portant sur le jeu, les jouets et les ludothèques ${ }^{7}$. L'ensemble des informations dorénavant disponibles à la Centrale des bibliothèques sur le thème du jeu pourrait en faire à long terme un centre privilégié d'information et de diffusion des thèmes de jeu. Dans le même cas, il est possible de regrouper les ludothèques, les joujouthèques et les services spécialisés dans le domaine du prêt de matériel de jeu, autour des services offerts par la Centrale.

En plus d'élargir constamment la collection d'analyses de jeux et de jouets, la Centrale se propose de diffuser ses travaux à l'étranger et surtout dans les pays francophones: il est également possible d'étendre le réseau d'information au-delà des limites mêmes de la Centrale par le biais d'un réseau de terminaux reliés à distance, à la base de données de la Centrale. Un tel réseau pourrait rendre possible la création de laboratoires de jeu et pourrait susciter de nouvelles recherches.
6. Le guide d'analyse des jouets de même que le fichier de définitions des descripteurs seront publiés au prıntemps 1983 à l'adresse suivante

Société du stage en Bıbliothéconomie

100. Avenue Paınchaud

La Pocatière-Québec-Canada

GOR 1 ZO
7. Rosaire Pelletier et Marielle Boucher, Le jeu et l'enfant, La Pocatière. La Centrale des bibliothèques et la Société du Stage en biblıothéconomie. 1982. 70 pages. 
En outre, la publication d'un guide d'indexation et d'analyse du matériel de jeu, de même que du fichier de définitions des concepts psychopédagogiques servant de support au thésaurus, permettra de former de nouveaux analystes du jeu selon les techniques et les méthodes d'analyse du modèle ESAR.

\section{Conclusion}

L'analyse systématique du matériel de jeu et de jouets est une nécessité dans le domaine de l'éducation. En effet, la surenchère qui a marqué la fabrication commercialisée des objets ludiques au cours des dernières décennies rend la tâche de plus en plus difficile pour les éducateurs si aucun principe ne vient orienter ce choix d'une manière efficace.

Le système ESAR, dans ce domaine, peut rendre de précieux services aux éducateurs professionnels et aux parents. II permet d'identifier l'inexistence de matériel ludique sous certaines catégories ou à l'inverse pointer du doigt certaines catégories ou sous-catégories trop fortement représentées sur le plan commercial au détriment de nouvelles formes de jeux possibles.

Le système ESAR présente donc au milieu de l'éducation et au monde des techniques documentaires, un instrument d'analyse psychopédagogique inédit, original, sur le développement de l'enfant; un instrument suffisamment cohérent et articulé pour susciter de nouvelles recherches et de multiples applications concrètes au cours des prochaines années, en plus de répondre à un besoin immédiat de classement, de catalogage. d'analyse et de choix de matériel ludique pour les services de prêt de jouets.

Denise Garon. Ph. D.

Techniques de garderie d'enfants

CEGEP de Ste-Foy

Québec

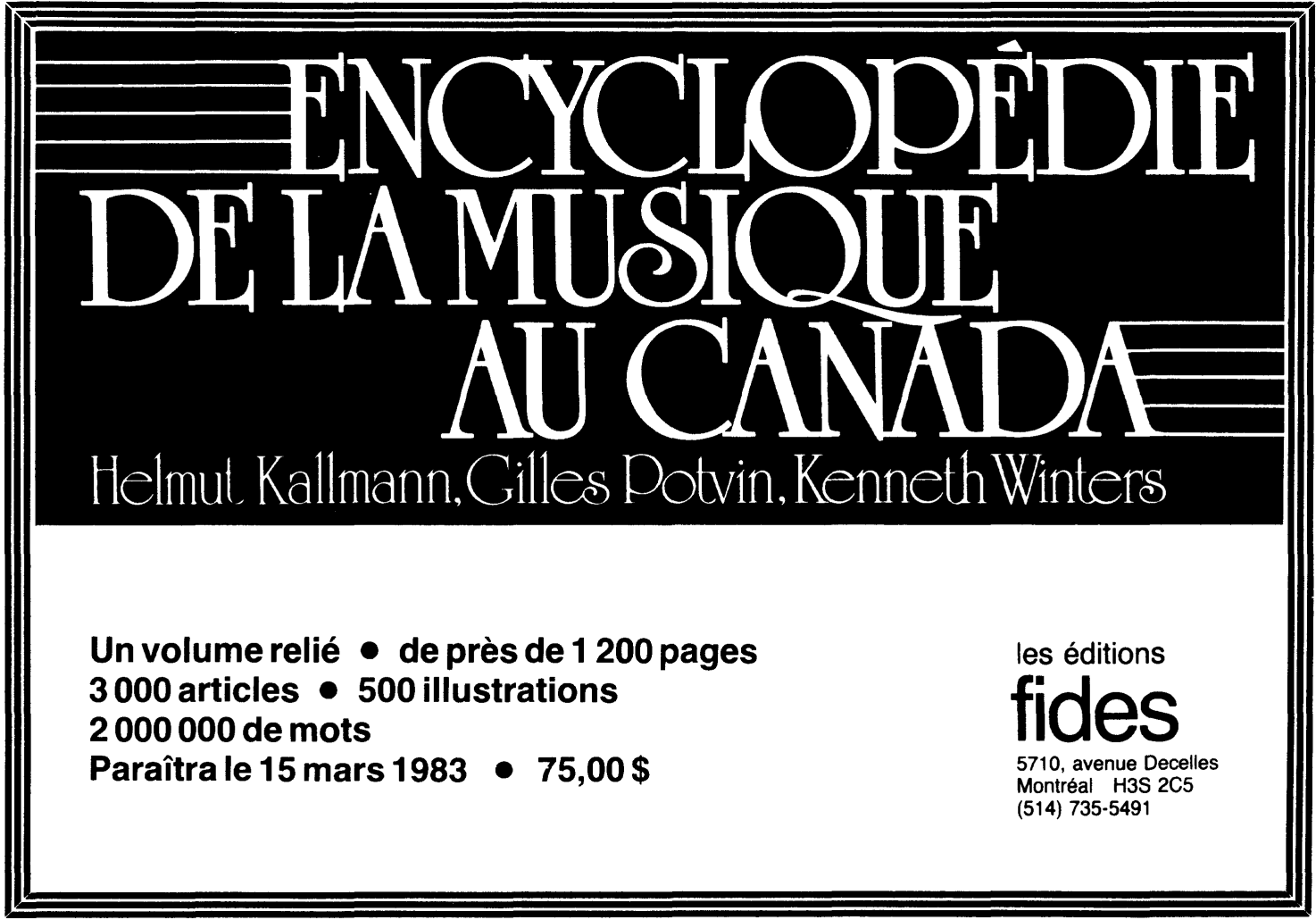

